\title{
High-Speed, Compact, Adaptive Lenses Using In- Line Transparent Dielectric Elastomer Actuator Membranes
}

\section{Citation}

Samuel Shian, Roger M. Diebold, and David R. Clarke. 2013. High-speed, compact, adaptive lenses using in-line transparent dielectric elastomer actuator membranes. In Proceedings of SPIE, Electroactive Polymer Actuators and Devices (EAPAD) 2013, ed. Yoseph Bar-Cohen, 8687: 86872D. Bellingham, Washington: Society of Photo-Optical Instrumentation Engineers (SPIE). Paper presented at Electroactive Polymer Actuators and Devices (EAPAD) XV, San Diego, CA, March 10-14, 2013.

\section{Published Version}

doi:10.1117/12.2009848

\section{Permanent link}

http://nrs.harvard.edu/urn-3:HUL.InstRepos:10579570

\section{Terms of Use}

This article was downloaded from Harvard University's DASH repository, and is made available under the terms and conditions applicable to Open Access Policy Articles, as set forth at http:// nrs.harvard.edu/urn-3:HUL.InstRepos:dash.current.terms-of-use\#OAP

\section{Share Your Story}

The Harvard community has made this article openly available.

Please share how this access benefits you. Submit a story.

\section{Accessibility}




\title{
High-speed, compact, adaptive lenses using in-line transparent dielectric elastomer actuator membranes
}

\author{
Samuel Shian*, Roger M. Diebold, David R. Clarke \\ School of Engineering and Applied Sciences, Harvard University, \\ Cambridge, Massachusetts 02138, USA
}

\begin{abstract}
Electrically tunable adaptive lenses provide several advantages over traditional lens assemblies in terms of compactness, speed, efficiency, and flexibility. We present an elastomer-liquid lens system which makes use of an in-line, transparent electroactive polymer actuator. The lens has two liquid-filled cavities enclosed within two frames, with two passive outer elastomer membranes and an internal transparent electroactive membrane. Advantages of the lens design over existing systems include large apertures, flexibility in choosing the starting lens curvature, and electrode encapsulation with a dielectric liquid. A lens power change up to 40 diopters, corresponding to focal length variation up to $300 \%$, was recorded during actuation, with a response time on the order of tens of milliseconds.
\end{abstract}

Keywords: Adaptive optics, focus tunable lens, dielectric elastomer actuators, acrylic elastomer, transparent electrodes

\section{INTRODUCTION}

Variable-focus or adaptive lenses are superior to traditional fixed-focus solid lens assemblies in terms of reduced bulk and weight as well as having a high-speed response, low material costs, and facile fabrication [1,2]. Such benefits can be used in imaging applications where space is at a premium, including endoscopes, cell phone cameras, and machine vision apparatus. Low-cost adaptive lenses with liquid components offer simple lens designs and the possibility of more economic manufacturing of phased arrays and larger lenses. A variety of actuation mechanisms are used to drive the deformation of liquid adaptive lenses, including rotating screws [1], electric motors [3], piezoelectric elements [4], electromagnetics [5], shape memory alloys [6], electrowetting [7], heating elements [8], ionic-polymers [9] or dielectric elastomer actuators (DEAs) [10]. However, each of these existing adaptive lens configurations locates the actuator outside the optical path. The primary consequence of placing the actuator outside the optical path is that a significant fraction of the lens' lateral area is occupied by the actuating mechanism, reducing the usable optical area. The use of transparent DEAs for lenses was recently demonstrated, but as the lens consists of a buckling solid elastomer membrane, it only operates as a diverging optic [11].

The demonstration of compliant, highly transparent, and electrically conductive electrodes in combination with dielectric elastomers affords the opportunity to use electrical signals to alter the shape of a liquid lens without external actuation [12]. The high optical transparency of the electrodes, liquid, and elastomer permits the construction of simple, selfcontained adaptive lenses suitable for space-constrained tasks. The purpose of this paper is to describe a self-contained, tunable lens with an integrated electrical actuator in the optical path of the lens. The two liquid cavity design allows electrode encapsulation with a dielectric liquid and the flexibility of choosing the lens curvature at rest. In this proceeding, a detailed description of the lens design, materials, and fabrication is presented. 


\section{LENS DESIGN}

The adaptive lens consists of two liquid cavities separated by a transparent dielectric elastomer actuator membrane. At rest, the liquid in each cavity is at a different hydrostatic pressure. The lens is fabricated from two stiff frames, one electroactive elastomer membrane, two passive elastomer membranes, and a transparent liquid of constant volume, as shown in Figure 1a. The stiff, relative to the elastomer, frames are concentric and have different diameters to define the cavities. The electroactive elastomer membrane is a transparent dielectric elastomer sheet coated with transparent compliant electrodes on both sides; in this case, the dielectric elastomer is a commercially available acrylic elastomer and the electrodes are single walled carbon nanotube (SWCNT) mats. This electroactive membrane is sandwiched between the stiff frames while the other sides of the frames are enclosed with the passive elastomer membranes, forming two cavities. One cavity is filled with transparent liquid at a higher pressure than ambient, or $P_{1}>P_{0}$, such that the adjacent membranes bulge outward. The other cavity is filled with the same transparent liquid but at a lower pressure than the first cavity, or $P_{2}<P_{1}$. Thus the curvature of the passive membrane in the first cavity will be convex while the curvature of the passive membrane in the second cavity can be convex $\left(P_{2}<P_{0}\right)$, flat $\left(P_{2}=P_{0}\right)$, or concave $\left(P_{2}>P_{0}\right)$, depending on the amount of fluid in the second cavity, as shown in Figure 1c.

(a)

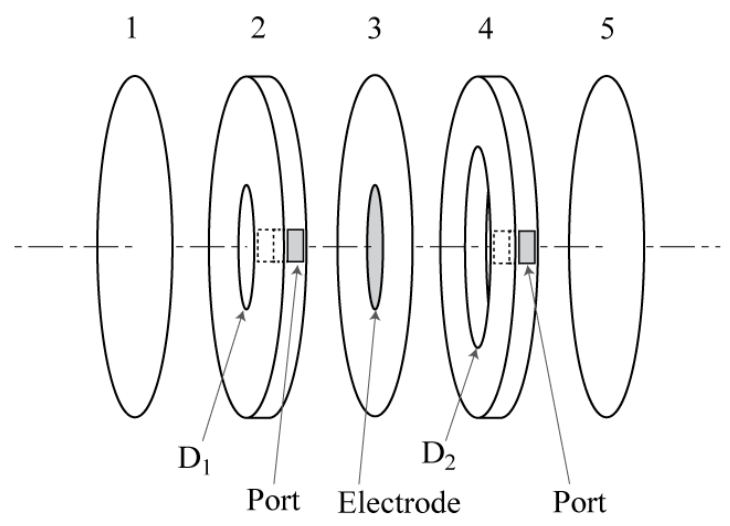

(c)

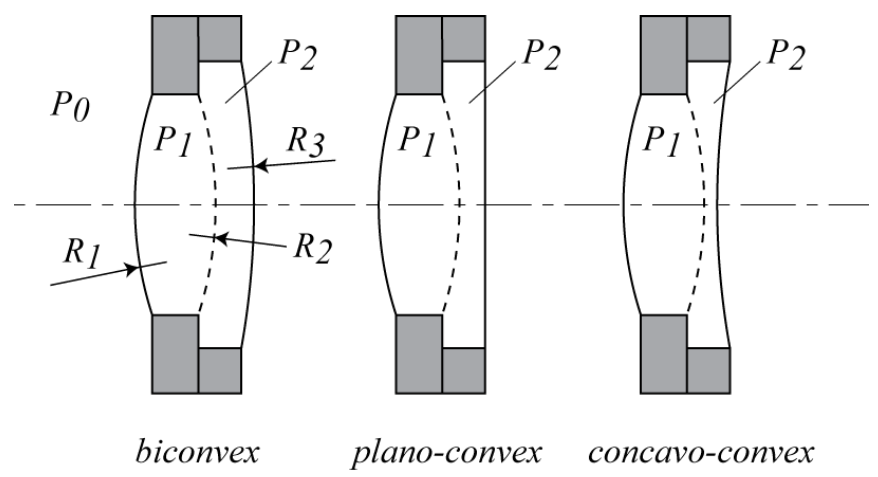
Port Electrode Port (b)

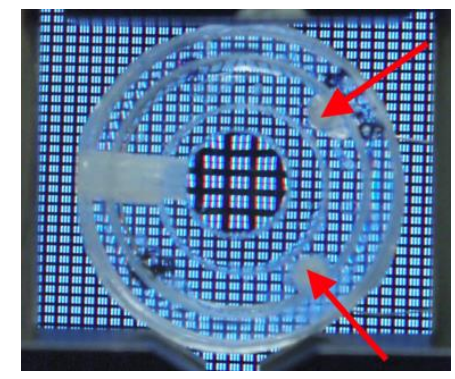


The lens' adaptive capability is driven by the relative curvature difference between the passive membranes as a function of the internal liquid pressure. When a voltage is applied to the DEA membrane, the Coulombic attraction between the charges generates a Maxwell stress over the membrane's thickness, causing it to stretch which, in turn, displaces volume in the plane of the electrodes. As the DEA deforms, the tension in the membrane decreases causing both a concomitant decrease and increase in liquid pressure in the first and second cavities, respectively. A unique feature of the lens is that the diameters of the passive membrane windows, $D_{1}$ and $D_{2}$, are not equal, allowing the change in curvature to differ between the two membranes, thus altering the focal length of the lens. To maximize the optically active area, which is defined by $D_{1}$, the larger membrane window, $D_{2}$, may be constructed as large as possible to the outer diameter of the lens frame, provided that the material of the frame is reasonably stiff and impermeable to the liquid. In comparison to the previously published lens design [13], this dual-cavity lens has several advantages, including the ability to operate one cavity at a sub-atmospheric pressure for concave lens applications, resistance to gravitational effects during a loss of DEA membrane tension affording reduced image distortion, and electrode encapsulation within the lens liquid.

\section{EXPERIMENTAL DETAILS}

The part numbering in the following text refers to the schematic shown in Figure 1a. A pair of lens frames was prepared from acrylic sheets ( $2 \mathrm{~mm}$ thickness, McMaster-Carr) cut into $25 \mathrm{~mm}$ outer diameter rings, each with inner concentric holes of $D_{1}=8 \mathrm{~mm}$ and $D_{2}=13 \mathrm{~mm}$, as shown in Figure 1a as parts 2 and 4, respectively. An injection port was drilled in each frame, later filled with a moisture-curing silicone elastomer (Silpoxy, Reynolds manufacturing). The passive elastomer membranes were made from transparent acrylic elastomer sheets (4905 or 4910,3M); the first membrane (part 1) is $0.5 \mathrm{~mm}$ thick and the second membrane (part 5) is $1 \mathrm{~mm}$ thick, which were linearly pre-stretched to $350 \%$ and $100 \%$, respectively. The DEA membrane was made from the same material as the first passive membrane, except SWCNT electrodes were deposited on both sides of the electroactive membrane using the filtration-transfer method described previously [12]. After bonding of the frame and membranes, clear silicone oil (Dow Corning Liquid 200, 50 cSt, refractive index 1.4022) was injected into the lens cavities through the injection ports.

For dynamic response measurements, a collimated white light was passed through the optical axis of the lens and collected by a fiber optic leading to a spectrophotometer (model USB 2000+, Ocean Optics). The detector was set at a 1 $\mathrm{ms}$ integration time, and the light intensity between 600 to $610 \mathrm{~nm}$ was measured as a function of time. When the lens was actuated, the focal length moved toward the fiber optic detector, increasing the density of light at the fiber optic tip and increasing the recorded light intensity.

\section{RESULTS}

The assembled lens is depicted in Figure 1(b) against a square grid background illustrating that most of the materials, including the frame, are optically transparent; the frames can be made opaque to prevent stray light if preferred. The acrylic DEA elastomer membranes have a high transparency as only a small fraction of light is lost through absorption. However, reflective light losses may be significant in the absence of an anti-reflective coating, which occurs primarily at the air-passive membrane interfaces. Inside the lens, such reflective losses are minimized since the refractive index of the acrylic membrane $(n=1.476)[14]$ and liquid $(n=1.4022)$ are similar. Thus, despite three membranes and two liquid mediums within the light path, the assembled lens has relatively high optical transmittance (> $80 \%$ at $550 \mathrm{~nm}$ ).

To visualize the focal length change when the DEA is electrically actuated, a laser beam was passed through the lens into water containing a dilute colloidal silica suspension (diameter $210 \mathrm{~nm}$, Bangs Lab.), as shown in Figure 2a. The silica particles were completely dispersed and slightly scatter the laser beam so that the beam shape can be captured photographically. Since the incoming laser beam was collimated, the light converges at the lens focal point, forming a cone. When an electrical bias is applied to the DEA electrodes, the lens focal length increases, as illustrated in the lower portion of the figure. 


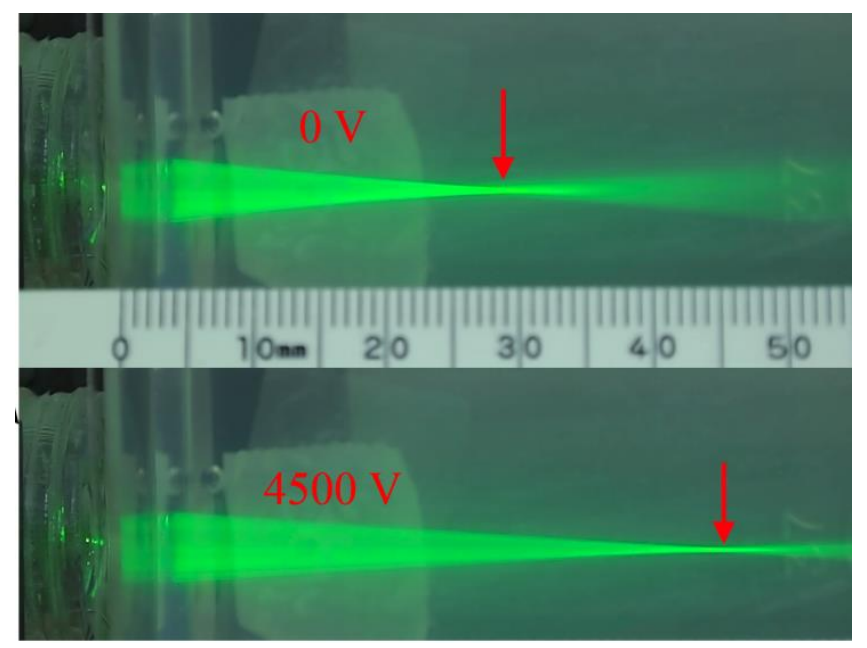

Figure 2. Photograph of the adaptive lens focal point (indicated by arrows), visualized using a green laser beam passed through a colloidal silica dispersion in water. The adaptive lens can be seen on the left side of the images. The upper and lower images show the focal point of the lens at $0 \mathrm{~V}$ and $4500 \mathrm{~V}$, respectively. The scale is in $\mathrm{mm}$.

The fabricated lens has a plano-convex configuration at rest, where the pressure in the first cavity is above ambient and equal to ambient in the second cavity. In this configuration, the composite focal length is determined by the amount of liquid in the first cavity. Increasing the amount of liquid in the first cavity decreases the lens curvature and subsequently reduces the initial focal length. Assuming spherical curvatures on the outer membranes, $R_{I}$ and $R_{3}$, the focal length of the lens, $f$, can be calculated using the thick lens equation,

$$
\frac{1}{f}=(n-1)\left[\frac{1}{R_{1}}-\frac{1}{R_{3}}+\frac{(n-1) d}{n R_{1} R_{3}}\right]
$$

where $n$ and $d$ are the refractive index of the liquid and the thickness of the lens, respectively. With no actuation, the curvature of the planar surface is infinity $\left(R_{3}=\infty\right)$ and the above equation can be simplified to $f=R_{l} /(n-1)$. For the focal length measurement test, the fabricated lens has an initial focal length of $15 \mathrm{~mm}$, corresponding to a lens power of 67 diopters. The calculated radius of curvature, $R_{l}$, and the apical height, $h$, of the hemisphere are 6 and $1.5 \mathrm{~mm}$, respectively. Since the ratio of $h /\left(D_{l} / 2\right)=0.37$ is less than 1 , the spherical cap geometry approximation is maintained [15]. During actuation, the DEA membrane stretches, increasing the curvature of the DEA membrane $\left(R_{2}\right)$, which increases $R_{1}$ and decreases $R_{3}$. The resulting net curvature change and the corresponding change in focal length originate from the relative difference in diameter $D_{2}$ with respect to $D_{1}$. As shown in our previous publication [13], the relative difference $D_{2} / D_{1}$ plays an important role in the amount of focal length change during actuation; the larger the ratio, the larger the dynamic focal length change. 


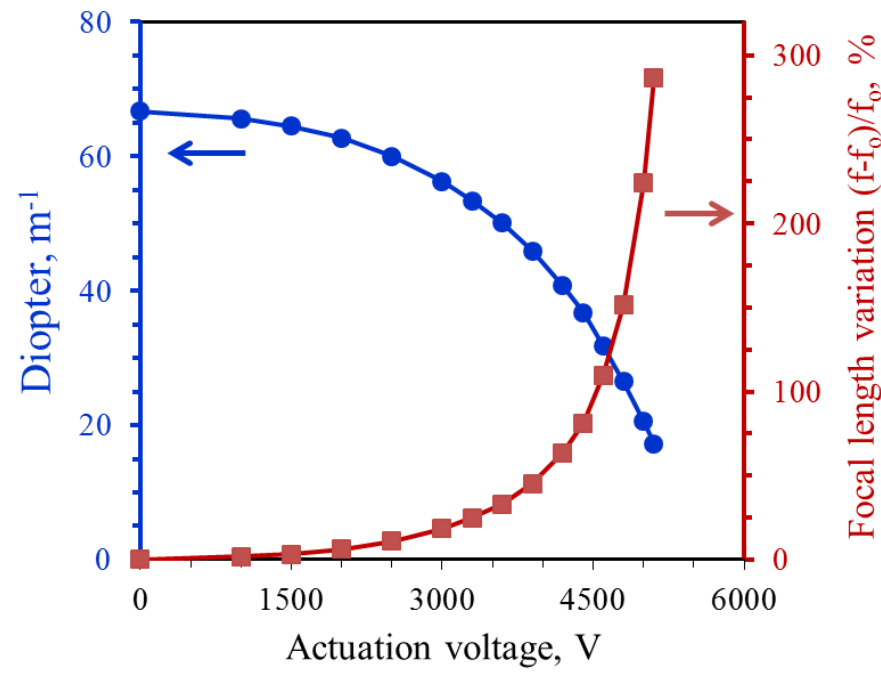

Figure 3. Lens power (diopter) and focal length variation as function of actuation voltage.

The diopter and the change in lens focal length with actuation voltage are shown in Figure 3. We did not observe image degradation due to non-concentric lens deformation at the highest actuation voltage, which is an improvement over the first lens design [13]. A tension loss or non-uniform deformation in the DEA membrane will not degrade the image since identical liquid fills both lens cavities and both the liquid and the acrylic membrane have similar refractive indices. The liquid pressure inside the first cavity drops monotonically with actuation strain [16], while the pressure inside the second cavity increases. In essence, the pressure from the first cavity is transferred to the second cavity during actuation. Since VHB is capable of very high actuation strains, on the order of hundreds of percent in area [17], the maximum lens focal length change is not limited by the actuation strain; rather, the maximum focal length is limited by the equilibrium curvature of the passive membranes which is achieved when the pressure in both cavities are equal, or $P_{1}=P_{2}$.

The fabricated lenses have response times of the order of tens of milliseconds, depending on the actuation strain or voltage. An example of the dynamic response of the lens when the input voltage was pulsed from $0 \mathrm{~V}$ to $4.5 \mathrm{kV}$ is shown in Figure $4 \mathrm{a}$. The rise and fall times from $0 \%$ to $90 \%$ relative intensity are 25 and 10 milliseconds, respectively, as shown by an analysis of a pulse response in Figure $4 \mathrm{~b}$. The rise time is characterized by a fast initial response followed by a slower rise, both of which are governed by the elastic and viscous properties of the acrylic elastomer, respectively. While DEAs can exhibit relatively fast response times in comparison to other electroactive polymer actuators $[17,18]$, the liquid's inertia and viscosity slows the response of the lens system; the more viscous, the longer the response time. Varying the appropriate lens parameters, such as decreasing the lens dimensions, using low density and low viscosity liquids, and employing elastomer membranes with high stiffness can further optimize the system for improved actuation speed. Additionally, improved DEA materials, with increased dielectric permittivity and reduced viscous losses would decrease the driving voltage for actuation and increase actuation speed, respectively.

Fast dynamic responses are particularly useful for close up photographic focus and image capture applications, where the adaptive lens is placed between a digital camera and relatively small objects, as shown in Figure 4c. In this demonstration, focus plane changes of $4 \mathrm{~mm}$ can be achieved in approximately $25 \mathrm{~ms}$ by switching the voltage between 0 and $3.9 \mathrm{kV}$. In traditional lens systems, such high-speed focusing requires high-powered electric motor and gear assemblies to move the lenses. Another focusing mechanism, moving the objects, is usually not feasible since the time and distance constraints correspond to linear acceleration of at least $2.6 \mathrm{~g}$, potentially causing undesirable vibrations and disturbing unfixed objects. The higher speed response of the adaptive lenses is a consequence of having to only displace the membrane rather than the entire lens and assembly.

The placement of a DEA membrane between two silicone oil cavities is expected to increase the reliability and lifetime of the adaptive lens since the electrodes are essentially encapsulated in the dielectric liquid. Such encapsulation of SWCNT electrodes has been shown to significantly increase the durability of DEA through the suppression of corona 
discharges [19]. These adaptive lenses are suitable for demanding applications such as those requiring constant or highly cyclic actuation over prolonged times, similar to that seen in Figure 4a.
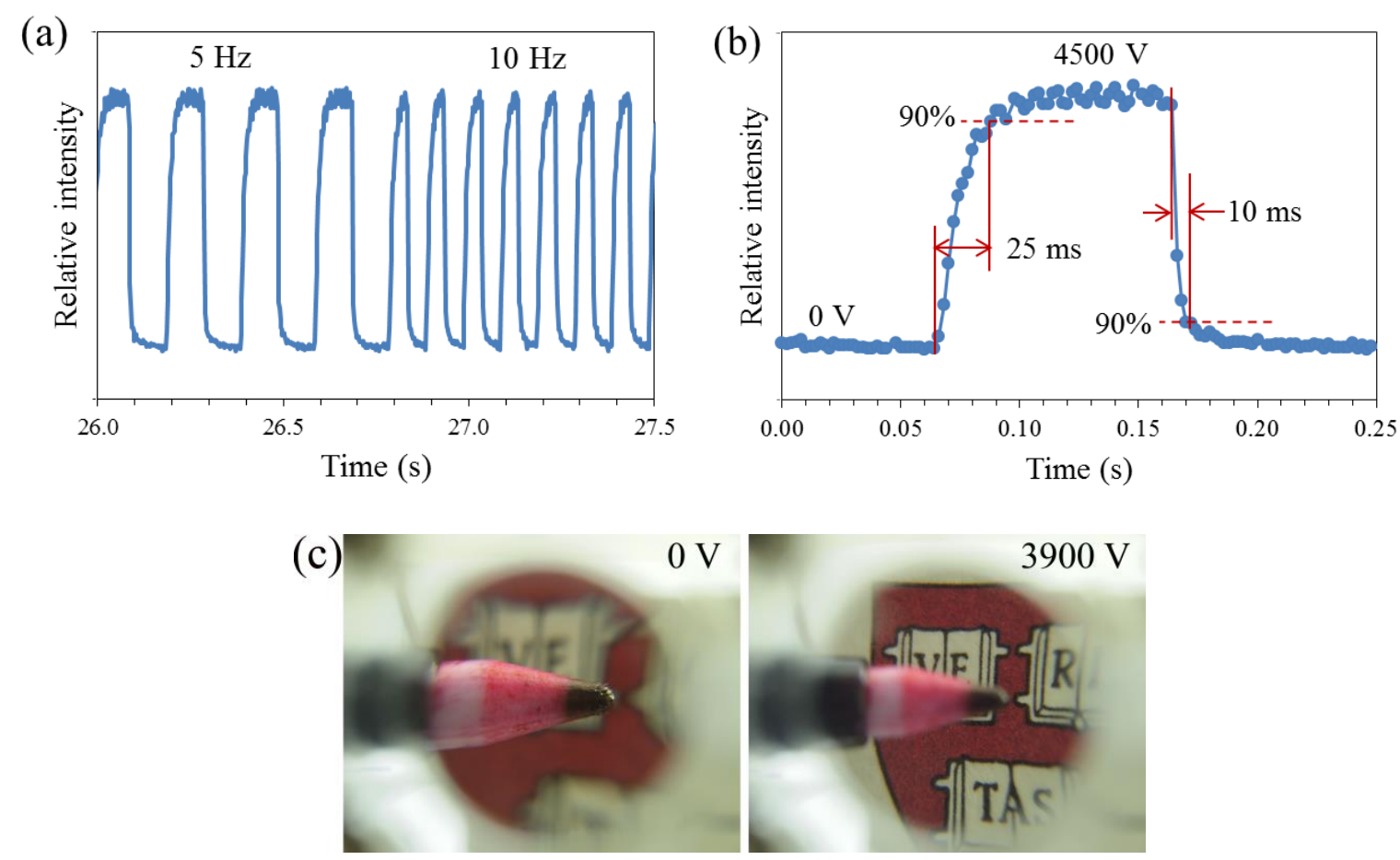

Figure 4. (a) The dynamic response of the lens when the actuation voltage is alternated between 0 to $4.5 \mathrm{kV}$ at a rate of 5 or $10 \mathrm{~Hz}$, as measured by spectrophotometric light intensity. (b) Higher magnification of one of the $5 \mathrm{~Hz}$ pulses. The response time of the lens is determined by the time required to reach $90 \%$ of the equilibrium intensity. (c) Close-up photographic images of a logo through the adaptive lens, used to increase the magnification of the camera. At rest, the lens is focused at the tip of a pen and upon actuation at $3.9 \mathrm{kV}$, the lens focus moves $4 \mathrm{~mm}$ towards the logo.

\section{CLOSING REMARKS}

Electrically tunable adaptive lenses have been demonstrated with a transparent actuator located directly in the optical path. Lenses built using this concept are capable of focal length changes of $300 \%$ at relatively fast response times, on the order of tens of milliseconds. Optimization of focal length change and lens response time can be achieved by choosing an appropriate liquid and lens geometry. Inherent encapsulation of the SWCNT electrodes by the silicone oil is expected to provide reliable operation and permit substantial lifetimes of the adaptive lens.

\section{ACKNOWLEDGMENTS}

The authors acknowledge the partial financial support by the Harvard MRSEC program of the National Science Foundation under Award number DMR-0820484

\section{REFERENCES}

[1] M. Blum, M. Büeler, C. Grätzel, and M. Aschwanden, "Compact optical design solutions using focus tunable lenses," in SPIE Proceedings Vol. 8167 (2011) [doi:10.1117/12.897608]. 
[2] Hongwen Ren and S.-T. Wu, Introduction to Adaptive Lenses, p. 286, John Wiley \& Sons, Inc., Hoboken, New Jersey (2012).

[3] H. Ren, D. Fox, P. A. Anderson, B. Wu, and S.-T. Wu, "Tunable-focus liquid lens controlled using a servo motor.," Optics express 14(18), 8031-8036 (2006).

[4] F. Schneider, J. Draheim, C. Müller, and U. Wallrabe, "Optimization of an adaptive PDMS-membrane lens with an integrated actuator," Sensors and Actuators A: Physical 154(2), 316-321 (2009) [doi:10.1016/j.sna.2008.07.006].

[5] P. Liebetraut, S. Petsch, W. Mönch, and H. Zappe, "Tunable solid-body elastomer lenses with electromagnetic actuation.," Applied optics 50(19), 3268-3274 (2011).

[6] H.-M. Son, M. Y. Kim, and Y.-J. Lee, "Tunable-focus liquid lens system controlled by antagonistic windingtype SMA actuator.," Optics express 17(16), 14339-14350 (2009).

[7] B. Berge and J. Peseux, "Variable focal lens controlled by an external voltage : An application of electrowetting," THE EUROPEAN PHYSICAL JOURNAL E 163, 159-163 (2000).

[8] W. Zhang, K. Aljasem, H. Zappe, and A. Seifert, "Completely integrated, thermo-pneumatically tunable microlens.," Optics express 19(3), 2347-2362 (2011).

[9] Y. Wang, H. Chen, B. Luo, and Z. Zhu, "Design and optimization of small-sized actuators for driving optical lens with different shapes based on IPMCs,” 83401I-83401I-7 (2012) [doi:10.1117/12.917413].

[10] F. Carpi, G. Frediani, S. Turco, and D. De Rossi, "Bioinspired Tunable Lens with Muscle-Like Electroactive Elastomers," Advanced Functional Materials 21(21), 4152-4158 (2011) [doi:10.1002/adfm.201101253].

[11] S. Son, D. Pugal, T. Hwang, H. R. Choi, J. C. Koo, Y. Lee, K. Kim, and J.-D. Nam, "Electromechanically driven variable-focus lens based on transparent dielectric elastomer.," Applied optics 51(15), 2987-2996 (2012).

[12] S. Shian, R. M. Diebold, A. McNamara, and D. R. Clarke, "Highly compliant transparent electrodes," Applied Physics Letters 101(6), 061101 (2012) [doi:10.1063/1.4742889].

[13] S. Shian, R. M. Diebold, and D. R. Clarke, “Tunable Lenses using Transparent Dielectric Elastomer Actuators," Submitted to Optical Express (2013).

[14] J. Risner, "Investigation of Dielectric Elastomer Actuation for Printable Mechatronics," p. 250, University of California, Berkeley with the University of California, San Francisco (2008).

[15] I. Jung, J. Xiao, V. Malyarchuk, C. Lu, M. Li, Z. Liu, J. Yoon, Y. Huang, and J. A. Rogers, "Dynamically tunable hemispherical electronic eye camera system with adjustable zoom capability," Proceedings of the National Academy of Sciences 108, 1788-1793 (2011).

[16] H. Wang, S. Cai, F. Carpi, and Z. Suo, "Computational Model of Hydrostatically Coupled Dielectric Elastomer Actuators," Journal of Applied Mechanics 79(3), 031008 (2012) [doi:10.1115/1.4005885].

[17] R. Pelrine, R. Kornbluh, Q. Pei, and J. Joseph, "High-Speed Electrically Actuated Elastomers with Strain Greater Than 100\%," Science 287(5454), 836-839 (2000) [doi:10.1126/science.287.5454.836]. 
[18] P. Brochu and Q. Pei, “Advances in dielectric elastomers for actuators and artificial muscles.," Macromolecular rapid communications 31(1), 10-36 (2010) [doi:10.1002/marc.200900425].

[19] W. Yuan, P. Brochu, S. M. Ha, and Q. Pei, "Dielectric oil coated single-walled carbon nanotube electrodes for stable, large-strain actuation with dielectric elastomers," Sensors and Actuators A: Physical 155(2), 278-284 (2009) [doi:10.1016/j.sna.2009.09.003]. 